\title{
Seletividade de herbicidas em pós-emergência e controle de plantas daninhas no gergelim
}

\section{Selectivity of post-emergent herbicides and weed control for sesame}

\author{
Samuel Freguglia Bereta \\ Instituto Federal Catarinense (IFC) - Campus Santa Rosa do Sul \\ E-mail: samuelbereta@hotmail.com \\ OrcID: https://orcid.org/0000-0002-6173-9015
}

\begin{abstract}
Marcos André Nohatto
Instituto Federal Catarinense (IFC) - Campus Santa Rosa do Sul

E-mail: marcos.nohatto@ifc.edu.br

OrclD: https://orcid.org/0000-0002-0820-330X
\end{abstract}

Eliete de Fátima Ferreira da Rosa Instituto Federal Catarinense (IFC) - Campus Santa Rosa do Sul

E-mail: eliete.rosa@ifc.edu.br OrclD: https://orcid.org/0000-0001-9131-81189

$\begin{array}{r}\text { Jéssica Fernandes Kaseker } \\ \text { Instituto Federal Catarinense (IFC) - Campus Santa Rosa do Sul } \\ \text { E-mail: jessica.kaseker@ifc.edu.br } \\ \text { OrclD: https://orcid.org/0000-0001-5487-7439 }\end{array}$

Mikael Cardoso dos Santos Instituto Federal Catarinense (IFC) - Campus Santa Rosa do Sul

E-mail: mikaelsantos@outlook.com OrclD: https://orcid.org/0000-0003-0130-9395

Ana Paula Werkhausen Witter Instituto Federal Catarinense (IFC) - Campus Santa Rosa do Sul E-mail: ana werkhausen@outlook.com OrcID: https://orcid.org/0000-0003-0524-5149

Resumo: A ampliação do gergelim (Sesamum indicum) no Brasil é dificultada por problemas relacionados ao manejo de plantas daninhas. Assim, o objetivo foi avaliar a seletividade de herbicidas aplicados em pósemergência no gergelim, bem como a resposta produtiva da cultura ao controle das plantas daninhas. Primeiramente, avaliou-se o comportamento do gergelim para diferentes herbicidas. Na sequência, a cultura foi submetida a diferentes doses do herbicida de maior seletividade (sethoxydim). Em campo, verificou-se o efeito das plantas daninhas sobre a cultura por meio dos tratamentos: testemunha, capinado e herbicida sethoxydim. As variáveis foram fitotoxicidade aos 7, 14, 21 e 28 dias após o tratamento (DAT), massa seca da parte aérea, altura de plantas, peso de mil sementes e produtividade. Imazethapyr, mesotrione, bentazon, carfentrazoneethyl e 2,4-D, quando aplicados em pós-emergência, não são foram seletivos para o gergelim. Sethoxydim foi seletivo entre 220,8 - 883,2 g i.a. ha-1, entretanto não proporcionou ganhos produtivos.

Palavras-chave: Fitotoxicidade. Sesamum indicum. Sethoxydim.

Abstract: The expansion of sesame (Sesamum indicum) sowing in Brazil is hampered by problems related to weed management. Therefore, the aim was to evaluate the selectivity of herbicides applied in post-emergence in sesame, as well as the productive response of the crop to weed control. First, the behavior of sesame for different herbicides was evaluated. Subsequently, the culture was subjected to different doses of the herbicide of greater selectivity (sethoxydim). In the field, the effect of weeds on the crop was verified through treatments: 
control, mechanical weeding and sethoxydim herbicide. The variables evaluated were phytotoxicity at $7,14,21$ and 28 days after treatment (DAT), shoot dry weight, plant height, the weight of one thousand seeds, and productivity. Imazethapyr, mesotrione, bentazon, carfentrazone-ethyl and 2,4-D, when applied in postemergence, are not selective for sesame. Sethoxydim was selective between doses $220.8-883.2 \mathrm{~g}$ a.i. ha ${ }^{-1}$, however it did not provide productive gains.

Keywords: Phytotoxicity. Sesamum indicum. Sethoxydim.

Data de recebimento: $22 / 04 / 2020$

Data de aprovação: 13/10/2020

DOI: https://doi.org/10.30612/agrarian.v14i51.11569

\section{Introdução}

O gergelim (Sesamum indicum) é uma planta oleaginosa pertencente à família Pedaliaceae, possuindo grande importância econômica no mundo (Perin et al., 2010). O Brasil ainda se caracteriza como pequeno produtor de gergelim, com 127 mil toneladas produzidas em 160 mil hectares. Contudo, constata-se o aumento no interesse do cultivo dessa cultura, destacando a evolução entre as safras 2018/2019 e 2019/2020, onde houve um incremento significativo de $307 \%$ e $302 \%$ na produção e área plantada, respectivamente (Conab, 2020).

A oleaginosa tem despertado interesse para exportação de sementes e derivados, dado a crescente demanda internacional, além de ser alternativa viável para o cultivo na safrinha, ao substituir o milho (Queiroga et al., 2012). O cultivo é destinado especialmente à produção de grãos para extração de óleo e outros produtos alimentícios, mas também tem nicho de mercado na indústria farmacêutica. Além disso, pode ser empregada como cultura-armadilha para mosca-branca e controle de formigas-cortadeiras (Ribeiro et al., 1998).

Essa cultura se insere nos sistemas tradicionais (convencional e plantio direto) e no sistema agroecológico. Pela tolerância à seca e pela facilidade de produção, destaca-se ainda pela opção de cultivo em sucessão, rotação e consorciação com outras culturas, o que desperta o interesse dos produtores que buscam alternativas de produção familiar (Perin et al., 2010).

A produtividade média do gergelim no Brasil é considerada muito baixa (796 kg ha-1) (Conab, 2020) diante da média obtida para a cultura em países como Líbano (3520 kg ha-1), Afeganistão $\left(2133 \mathrm{~kg} \mathrm{ha}^{-1}\right)$ e Israel $\left(2027 \mathrm{~kg} \mathrm{ha}^{-1}\right)$ (Faostat, 2018). Dentre os fatores responsáveis por esse desempenho destaca-se a interferência causada pelas plantas daninhas, pois o lento crescimento inicial do gergelim faz com que a cultura apresente menor habilidade competitiva na disputa pelos recursos do meio com as invasoras, prejudicando o seu potencial produtivo (Mane et al., 2017). O manejo mecânico tem sido a principal estratégia de controle, mesmo com uma série de limitações como baixo rendimento, maior dificuldade de eliminação das daninhas na linha de plantio e eventuais danos causados às raízes superficiais da cultura (Silva et al., 1999), sendo que tais medidas são adotadas, em grande parte, devido à ausência de herbicidas registrados para utilização dentro da cultura (Agrofit, 2020).

No que se refere ao controle químico, estudos indicam que a utilização em pré-emergência dos herbicidas acetochlor (1,26 kg i.a. ha $\left.{ }^{-1}\right)$ e S-metolachlor (1,43 kg i.a. ha $\left.{ }^{-1}\right)$ apresentam leve injúria $(0$ a $1 \%)$ sobre o gergelim (Grichar et al., 2018). Langham et al. (2007) apontam que os herbicidas diuron, fluchloralin, flumeturon, linuron, pendimethalin e trifluralin são seletivos para a cultura, mas novamente sobre uso em préemergência. Com interesse de ampliar as alternativas de manejo de plantas daninhas após a implantação da cultura, favorecendo a obtenção de maiores produtividades, foi realizado um estudo com o objetivo de avaliar a seletividade de herbicidas aplicados em pós-emergência no gergelim, bem como a resposta produtiva da cultura ao controle das plantas daninhas.

\section{Material e Métodos}

Três experimentos foram realizados no Instituto Federal Catarinense Campus Santa Rosa do Sul/SC, ano agrícola 2018/2019, sendo dois em casa-de-vegetação e outro a campo. A avaliação dos atributos químicos e porcentagem de argila do solo foi realizada antes da instalação dos experimentos, conforme metodologia proposta por Embrapa (2009), a fim de embasar as recomendações de adubação e manejo (Tabela 1). 
Tabela 1. Análise química e porcentagem de argila de um Gleissolo Melânico, com textura arenosa.

\begin{tabular}{|c|c|c|c|c|c|c|c|c|c|}
\hline $\begin{array}{c}\text { Argila } \\
\%\end{array}$ & $\mathrm{pH} \mathrm{H} \mathrm{H}_{2} \mathrm{O}$ & SMP & $\begin{array}{c}\text { M.O. } \\
\%\end{array}$ & \multicolumn{2}{|c|}{$\mathrm{mg} \mathrm{dm}^{-3}$} & \multicolumn{4}{|c|}{$\begin{array}{l}\mathrm{V} \\
\% \\
\end{array}$} \\
\hline 15 & 6,2 & 6,3 & 2,5 & 76,1 & 61,0 & \multicolumn{4}{|c|}{71} \\
\hline $\mathrm{Al}$ troc. & $\mathrm{Ca}$ troc. & $\mathrm{Mg}$ troc. & $\mathrm{H}+\mathrm{Al}$ & СТC & S & $\mathrm{Zn}$ & $\mathrm{Cu}$ & B & $\mathrm{Mn}$ \\
\hline \multicolumn{5}{|c|}{$\ldots \cdots \cdots \cdots \cdots \cdots \cdots \cdots \cdots \mathrm{cmol}_{\mathrm{c}} \mathrm{dm}^{-3} \cdots \cdots \cdots \cdots \cdots \cdots \cdots$} & \multicolumn{5}{|c|}{$\cdots \cdots \cdots \cdots \cdots \cdots \cdots \mathrm{mg} \mathrm{dm}^{-3} \cdots \cdots \cdots \cdots$} \\
\hline 0 & 5,1 & 2,2 & 3,1 & 7,5 & 16,7 & 5,7 & 3,5 & 0,31 & 9,5 \\
\hline
\end{tabular}

pH em água. SMP: pH medido após adição de solução tamponada SMP. M.O.: Matéria orgânica = C.org x 1,724. P, K, Zn, Cu e Mn: extrator Mehlich-1. Ca, Mg e Al: extrator $\mathrm{KCl}\left(1 \mathrm{~mol} \mathrm{~L}^{-1}\right)$. H+Al: extrator acetato de cálcio $\left(0,5 \mathrm{~mol} \mathrm{~L}^{-1}\right)$. V: Saturação de bases. CTC: capacidade de troca de cátions. B: extraído com água quente. S: extrator $\mathrm{Ca}\left(\mathrm{H}_{2} \mathrm{PO}_{4}\right)_{2} \cdot \mathrm{H}_{2} \mathrm{O}\left(500 \mathrm{mg} \mathrm{L}^{-1}\right.$ de $\left.\mathrm{P}\right)$.

Para os dois experimentos iniciais, o delineamento experimental foi inteiramente casualizado com cinco repetições. As sementes de gergelim (cultivar IAC Ouro) foram semeadas em vasos plásticos, devidamente perfurados, com capacidade volumétrica para $1 \mathrm{~L}$, contendo solo peneirado classificado como GLEISSOLO MELÂNICO (Santos et al., 2018). Após a emergência e estabelecimento das plântulas, foi realizado desbaste, deixando-se 2 plantas por unidade experimental.

Os tratamentos referentes ao primeiro experimento foram: sethoxydim $(220,8 \mathrm{~g}$ i.a. ha-1 $)$, imazethapyr

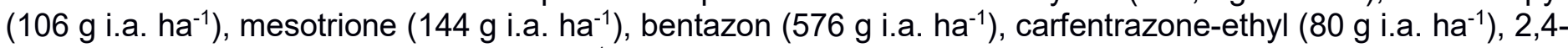
D (670 gramas de equivalente ácido ha-1) e testemunha (sem aplicação). Os tratamentos herbicidas foram pulverizados em pós-emergência, quando as plantas estavam no estádio de quatro folhas. Com exceção do 2,4-D, para os demais herbicidas utilizou-se adjuvante não iônico $(0,5 \% \mathrm{v} / \mathrm{v})$ no preparo da calda. Para aplicação, empregou-se pulverizador costal elétrico, equipado com pontas tipo leque AD 110.015, distribuindose volume de calda equivalente a $150 \mathrm{~L} \mathrm{ha}^{-1}$ e velocidade de deslocamento de $3,6 \mathrm{~km} \mathrm{~h}^{-1}$. Os vasos foram mantidos com irrigação diária, de forma a manter a umidade na capacidade de campo, ajustando o conteúdo de água por meio de pesagens.

Aos 7, 14, 21 e 28 dias após o tratamento (DAT) foi avaliado a fitotoxicidade dos herbicidas visualmente utilizando-se escala percentual, em que zero representou ausência de sintomas e 100 a morte das plantas (Velini et al.,1995). Aos 28 DAT, as plantas foram cortadas rente ao solo para a obtenção da massa seca da parte aérea (MSPA). Para isso, foram acondicionadas em estufa de circulação de ar quente a $60^{\circ} \mathrm{C}$ por período de 72 horas. Os resultados obtidos foram submetidos à análise de variância $(p \leq 0,05)$. No caso de ser constatada significância estatística, foi realizada a comparação de médias pelo teste Tukey $(p \leq 0,05)$.

$O$ herbicida que apresentou menor fitotoxicidade sobre a cultura, identificado com base nos resultados provenientes do primeiro experimento, foi utilizado para o segundo estudo. Nesse, seguindo as mesmas características do anterior (unidade, delineamento experimental, época de aplicação e análise estatística), foram aplicadas doses do herbicida selecionado ( $0,1 X, 2 X, 3 X$ e $4 X)$, sendo $X$ a dose padrão utilizada no experimento 1, avaliado fitotoxicidade e massa seca de parte aérea das plantas de gergelim aos 28 DAT.

Para o experimento realizado a campo (terceiro estudo) adotou-se delineamento em blocos casualizados e quatro repetições. Dados climáticos de precipitação e temperatura média diárias do período de condução do experimento foram fornecidas pela Epagri/Ciram (2020). Cada parcela foi constituída de 1,6 m de largura e $2 \mathrm{~m}$ de comprimento (área 3,2 $\mathrm{m}^{2}$ ), sendo realizado a semeadura em espaçamento de 0,40 $\mathrm{m} \times 0,20$ $\mathrm{m}$ entre linhas e plantas, respectivamente, totalizando $125 \mathrm{mil}^{\text {plantas ha-1 }}{ }^{-1}$ No preparo da área experimental, foi realizada dessecação das plantas daninhas com herbicida glyphosate (1080 g e.a. ha- $\left.{ }^{-1}\right)$. Após três semanas, o solo foi revolvido com enxada rotativa, sendo também realizada a abertura de sulcos para semeadura. 


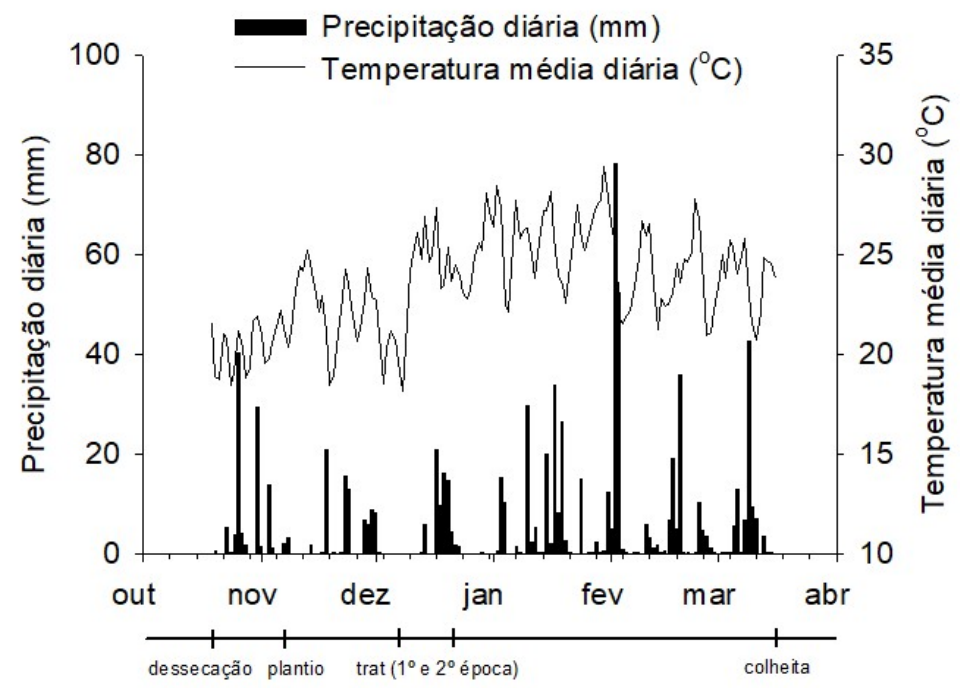

Figura 1. Precipitação pluvial diária $(\mathrm{mm})$ e temperatura média diária $\left({ }^{\circ} \mathrm{C}\right)$ ao longo do período experimental (Epagri/Ciram, 2020), indicando a época da realização das seguintes práticas: dessecação da área, plantio, aplicação dos tratamentos e colheita do gergelim.

Após a emergência, foi realizado desbaste no campo, a fim de manter a população estabelecida. $O$ estudo foi composto de três tratamentos: testemunha; capinado; sethoxydim (220,8 g i.a. ha-1). A aplicação do herbicida e o número de capina foram realizadas em duas épocas (estádio de 4 e 12 folhas da cultura), prática baseada em orientações de Arriel et al. (2007). Posteriormente, aos 14 dias após a última aplicação dos tratamentos, foram efetuadas amostragens das plantas daninhas nas parcelas a fim de realizar a avaliação das principais espécies presentes na área e obtenção da massa seca das plantas daninhas. Para isso, foi disposto aleatoriamente um quadro vazado de madeira com 0,4 $\mathrm{m}$ de lado e área interna de 0,16 $\mathrm{m}^{2}$, sendo uma repetição por parcela (Witter et at., 2019).

Aos 120 dias após a emergência, as avaliações para a cultura foram: altura de plantas $(\mathrm{m})$, obtida pela mensuração da distância da base até o ápice do meristema apical; produtividade $\left(\mathrm{kg} \mathrm{ha}^{-1}\right)$, pesagem dos grãos obtida de 5 plantas coletadas aleatoriamente dentro da unidade experimental; e peso de mil sementes (gramas), determinada por cinco amostras de 1000 sementes de cada tratamento, pesadas em balança de precisão $(0,0001 \mathrm{~g})$. Para as plantas daninhas foi avaliado a massa seca da parte aérea (gramas) total de plantas presentes no quadro vazado lançado no interior da parcela. Os dados obtidos foram submetidos à análise de variância $(p \leq 0,05)$. No caso de ser constatada significância estatística, foi realizada a comparação de médias entre os tratamentos pelo teste Tukey $(p \leq 0,05)$.

\section{Resultados e Discussão}

Para o primeiro experimento, observou-se que os herbicidas bentazon, mesotrione, 2,4-D, carfentrazone-ethyl apresentaram elevada injúria à cultura caracterizado pela necrose, essa já foi constatada nas primeiras avaliações aos 7 dias após a aplicação dos tratamentos (DAT), sendo a média de fitotoxicidade acima de $75 \%$ (Tabela 2). Os herbicidas sethoxydim e imazethapyr foram os que apresentaram menor fitotoxicidade na primeira época de avaliação, observando-se sintomas de clorose foliar nas regiões meristemáticas para o imazethapyr, inibidor da ALS (Acetolactato sintase).

Nas demais avaliações de fitotoxicidade, constatou-se aumento da fitotoxicidade para todos os herbicidas, com exceção do sethoxydim (Tabela 2). Verificou-se também que o imazethapyr não apresentou $100 \%$ de injúria nas plantas, porém mostrou elevada ação fitotóxica sobre a cultura (84\%) aos 28 DAT, na dose avaliada do estudo, que o classifica como não seletivo para o gergelim. 
Tabela 2. Fitotoxicidade e massa seca da parte aérea (MSPA) na cultura do gergelim após a aplicação dos tratamentos.

\begin{tabular}{llllll}
\hline Tratamento & 7 DAT & 14 DAT & 21 DAT & 28 DAT & MSPA \\
\cline { 2 - 6 } & \% & & & & (g) \\
\hline Testemunha & $0,0 \mathrm{e}^{1}$ & $0,0 \mathrm{~d}$ & $0,0 \mathrm{c}$ & $0,0 \mathrm{c}$ & $0,778 \mathrm{a}$ \\
Sethoxydim & $6,0 \mathrm{e}$ & $0,8 \mathrm{~d}$ & $0,8 \mathrm{c}$ & $0,4 \mathrm{c}$ & $0,715 \mathrm{a}$ \\
2,4-D & $100,0 \mathrm{a}$ & $100,0 \mathrm{a}$ & $100,0 \mathrm{a}$ & $100,0 \mathrm{a}$ & $0,132 \mathrm{~b}$ \\
Carfentrazone- & $100,0 \mathrm{a}$ & $100,0 \mathrm{a}$ & $100,0 \mathrm{a}$ & $100,0 \mathrm{a}$ & $0,075 \mathrm{~b}$ \\
ethyl & $78,0 \mathrm{c}$ & $82,5 \mathrm{~b}$ & $84,3 \mathrm{ab}$ & $100,0 \mathrm{a}$ & $0,053 \mathrm{~b}$ \\
Bentazon & $50,0 \mathrm{~d}$ & $67,0 \mathrm{c}$ & $75,0 \mathrm{~b}$ & $84,0 \mathrm{~b}$ & $0,120 \mathrm{~b}$ \\
Imazethapyr & $90,0 \mathrm{~b}$ & $100,0 \mathrm{a}$ & $100,0 \mathrm{a}$ & $100,0 \mathrm{a}$ & $0,091 \mathrm{~b}$ \\
Mesotrione & 6,6 & 7,8 & 13,1 & 9,3 & 22,3 \\
\hline $\mathrm{CV}^{2}(\%)$ & 6,6 &
\end{tabular}

${ }^{1}$ Médias seguidas pela mesma letra nas colunas, não diferem entre si pelo teste de Tukey $(\mathrm{p} \leq 0,05) .{ }^{2}$ Coeficiente de variação. ${ }^{3}$ Dias após aplicação dos tratamentos.

Ainda para o primeiro experimento, ao avaliar o comportamento da variável MSPA aos 28 DAT resultante da aplicação dos herbicidas, reforça-se a seletividade do sethoxydim, uma vez que tal herbicida não diferiu da testemunha (Tabela 2). Semelhantemente ao que ocorreu com o gergelim, outras culturas em expansão como cártamo (Carthamus tinctorius), grão de bico (Cicer arietinum L.) e feijão-caupi (Vigna unguiculata) também permite-se a utilização em pós-emergência do sethoxydim por atuar no controle de plantas daninhas, apresentando seletividade para a cultura (Castro et al., 2017; Araújo et al., 2019; Pereira et al., 2020).

Estudos realizados com propaquizafop e quizalofop (Mruthul et al., 2015; Ambika \& Sundari, 2019), herbicidas também inibidores da ACCase, demonstram seletividade para a cultura do gergelim, corroborando com os dados obtidos. A tolerância do gergelim a esse grupo de herbicidas possivelmente deve-se a presença de duas formas da enzima ACCase na planta, homodimérica no citosol e outra heterodimérica nos plastídeos. Os herbicidas inibidores da ACCase atuam somente sobre a forma homodimérica que está presente nos cloroplastos, impedindo a síntese de ácidos graxos nessa organela das gramíneas, não tendo atividade sobre a forma heterodimérica presente nas eudicotiledôneas (Carvalho, 2013).

No segundo experimento, os aumentos da dose de sethoxydim não provocaram redução da massa seca das plantas de gergelim, evidenciando a seletividade deste herbicida para esta cultura (Figura 2). Ainda, não houve ajuste às funções matemáticas testadas para a variável massa seca da parte aérea.

O conhecimento sobre o comportamento das plantas de gergelim a herbicidas em pós-emergência é restrito a poucos estudos, mesmo considerando a importância do tema para cultura. Imoloame et al. (2010) e Magani et al. (2012) utilizaram diferentes doses do fluazifop-p-butil no controle de plantas daninhas no gergelim e não observaram efeitos fitotóxicos na cultura pelo herbicida também pertencente ao grupo químico dos inibidores da ACCase, o que corrobora com os dados encontrados nessa pesquisa.

Para o terceiro experimento, identificou-se na amostragem que as plantas daninhas de espécies de maior ocorrência na área experimental foram do gênero Echinochloa, Cyperus e Ipomoea, pertencentes as Famílias Poaceae, Cyperaceae e Convovulaceae, respectivamente. O capim-arroz (Echinochloa sp.) e a tiririca (Cyperus sp.) por apresentarem metabolismo de planta C4, apresentam eficiência da fotossíntese sob altas temperaturas e luminosidade, condições frequentemente identificadas para a região, durante a época de cultivo, consequentemente, tal característica confere para as daninhas citadas vantagens competitivas sobre a cultura do gergelim, pertencente ao ciclo fotossintético C3.

Para as variáveis altura, produtividade e peso de mil sementes observou-se maiores valores para o tratamento com capina, demonstrando a influência negativa que a interferência das plantas daninhas causou sobre o desempenho do gergelim (Tabela 3). Considerando apenas a comparação entre a testemunha e capina, detectou-se redução para a variável altura $(\mathrm{m})$, produtividade $\left(\mathrm{kg} \mathrm{ha}^{-1}\right)$ e peso de mil sementes $(\mathrm{g})$ de $32,5 \%$, $90,8 \%$ e $11,7 \%$, respectivamente. 


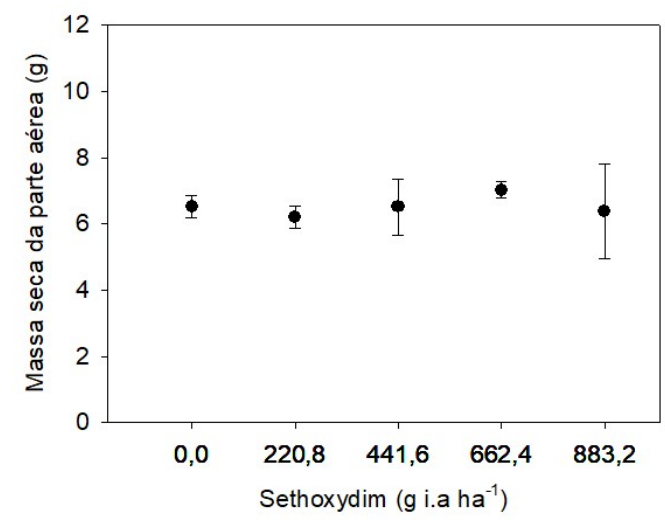

Figura 2. Massa seca da parte área de plantas de gergelim após a aplicação de doses de sethoxydim (0; 220,8; 441,$6 ; 662,4$ e 883,2 g i.a. ha $^{-1}$ ) com desvio-padrão.

Bhadauria et al. (2012) e Shaalan et al. (2014) também mostraram grande impacto das plantas daninhas sobre o gergelim, reduzindo $75 \%$ e $81 \%$ da produtividade na cultura, respectivamente. Porém, ljlal et al. (2011) apontam valores menores de decréscimo entre 6,88 a 12,40\% com seis semanas de competição com a cultura. A variação entre autores deve-se possivelmente as condições do ambiente experimental, como banco de sementes de plantas daninhas presentes na área de cultivo, condições edafoclimáticas do local e habilidade competitiva das cultivares. Lins et al. (2019) ao avaliarem duas cultivares de gergelim em dois anos quanto ao período crítico de prevenção a interferência, demonstraram que o controle de plantas daninhas é dependente da habilidade competitiva da cultivar, pois verificaram que para a cultivar BRS Seda o manejo deve iniciar entre o $12^{\circ}$ e $15^{\circ}$ dias após a emergência (DAE), enquanto para a CNPA G2 entre o $17^{\circ}$ e $20^{\circ} \mathrm{DAE}$, alcançando perda de 2,5 e $5,0 \%$, respectivamente.

Tabela 3. Altura, produtividade e peso de mil sementes de gergelim com ou sem aplicação de sethoxydim $(220,8$ $\mathrm{g}$ i.a. ha $\left.{ }^{-1}\right)$; e massa seca da parte aérea de plantas daninhas $(\mathrm{g})\left(0,16 \mathrm{~m}^{2}\right)$, avaliado aos 120 dias após a emergência.

\begin{tabular}{llcll}
\hline Tratamento & $\begin{array}{l}\text { Altura de plantas } \\
(\mathbf{m})\end{array}$ & $\begin{array}{l}\text { Produtividade } \\
\mathbf{( k g ~ h a}^{-1} \mathbf{)}\end{array}$ & $\begin{array}{l}\text { Peso de mil } \\
\text { sementes } \mathbf{( g )}\end{array}$ & $\begin{array}{l}\text { MSPA } \\
(\mathbf{g} \text { - daninhas })\end{array}$ \\
\hline Testemunha & $0,87 \mathrm{~b}^{1}$ & $109,74 \mathrm{~b}$ & $2,64 \mathrm{~b}$ & $81,10 \mathrm{a}$ \\
Capina & $1,29 \mathrm{a}$ & $1188,42 \mathrm{a}$ & $2,99 \mathrm{a}$ & $0,00 \mathrm{c}$ \\
Sethoxydim & $0,88 \mathrm{~b}$ & $210,17 \mathrm{~b}$ & $2,50 \mathrm{~b}$ & $35,47 \mathrm{~b}$ \\
\hline CV $(\%)$ & 8,5 & 18,6 & 5,5 & 21,3 \\
\hline
\end{tabular}

${ }^{1}$ Médias seguidas pela mesma letra nas colunas, não diferem entre si pelo teste de Tukey $(p \leq 0,05)$.

Embora o uso de sethoxydim tenha levado a reduções na massa seca das plantas daninhas se comparado a testemunha, seu uso ainda não foi eficaz para atingir altos níveis produtivos (Tabela 3). Por controlar apenas plantas daninhas de folhas estreitas, o uso de sethoxydim foi eficaz apenas para essas espécies. Portanto, para controlar espécies eudicotiledóneas, o uso de sethoxydim deveria ser acompanhando de um herbicida com outro mecanismo de ação.

Além da competição proporcionada por plantas daninhas eudicotiledóneas, a presença delas na área, como por exemplo, a corda-de-viola, potencializa o prejuízo, pois é uma planta trepadeira, que se enrola sob o caule e folhas do gergelim, prejudicando ainda mais o desenvolvimento da cultura. Assim, em áreas onde o banco de sementes e histórico revelarem alta incidência de plantas daninhas de folhas largas, outras estratégias de controle deverão ser intensificadas, a fim de reduzir a mato-competição proporcionada por essas invasoras.

\section{Conclusões}

Os herbicidas imazethapyr, mesotrione, bentazon, carfentrazone-ethyl e 2,4-D, quando aplicados em pós-emergência, não são foram seletivos para o gergelim. O uso de sethoxydim foi seletivo para o gergelim entre as doses $220,8 \mathrm{~g}$ i.a. ha-1 e $883,2 \mathrm{~g}$ i.a. ha-1, entretanto não proporcionou ganhos produtivos. 


\section{Referências}

Agrofit - Sistema de agrotóxicos fitossanitários. (2020). Disponível em: http://agrofit.agricultura.gov.br/agrofit cons/principal agrofit cons. Acesso em: 20 abril 2020.

Ambika, M. \& Sundari, A. (2019). Weed management in irrigated sesame (Sesamum indicum L.). World Scientific News, 131(1), 272-278.

Araújo, L.S., Silva, L.G.B., Valente, M.S., Silva, A.R., Cunha, P.C.R., Nascimento, W.M. (2019). Selectivity of post-emergence herbicides for the chickpea. Journal of Agricultural Science, 11(18), 179-186. https://doi.org/10.5539/jas.v11n18p179

Arriel, N.H.C., Firmino, P.T., Beltrão, N.E.M., Soares, J.J., Araújo, A.E., Silva, A.C., Ferreira, G.B. (2007). A cultura do gergelim. Brasília: Embrapa Informação Tecnológica.

Bhadauria, N.; Arora, A., Yadav, K.S. (2012). Effect of weed management practices on seed yield and nutrient uptake in sesame. Indian Journal of Weed Science, 44(2), 129-131. https://doi.org/10.5958/0976$\underline{0571.2015 .00136 .8}$

Carvalho, L.B. (2013). Herbicidas. Lages: Autor.

Castro, E.B., Belapart, D., Carbonari, C.A., Zanotto, M.D., Ben, R., Macedo, G.C., Simões, P.S. (2017). Seletividade de herbicidas aplicados em pré e pós-emergência da cultura do cártamo. Revisa Brasileira de Herbicidas, 16(3), 230-237. https://doi.org/10.7824/rbh.v16i3.518

CONAB - Companhia Nacional de Abastecimento. Acompanhamento da safra brasileira de grãos. (2020). Disponível em: https://www.conab.gov.br/info-agro/safras/graos/boletim-da-safra-de-graos. Acesso em: 21 abril 2020.

Embrapa - Embrapa Solos. (2009). Manual de análises químicas de solos e fertilizantes. Brasília: Embrapa Solos.

EPAGRI/CIRAM - Empresa de Pesquisa Agropecuária e Extensão Rural de Santa Catarina/ Centro de Informações de Recursos Ambientais e de Hidrometeorologia de Santa Catarina. Disponível em: http://ciram.epagri.sc.gov.br/index.php?option=com content\&view=featured\&ltemid=101. Acesso em: 18 junho 2020.

Faostat - Food and Agriculture Organization of the United Nations (FAO). FAOSTAT Database. (2018). Disponível em: http://www.fao.org/faostat/en/\#data/QC. Acesso em: 17 junho 2020.

Grichar, W.J., Rose, J.J., Dotray, P.A., Baughman, T.A., Langham, D.R., Werner, K., Bagavathiannan, M. (2018). Response of sesame to selected herbicides applied early in the growing season. International Journal of Agronomy, 2018(1), 1-11. https://doi.org/10.1155/2018/9373721

ljlal, Z., Tanveer, A., Safdar, M.E., Aziz, A., Ashraf, M., Akhtar, N., Atif, F.A., Ali, A., Maqbool, M.M. (2011). Effects of weed crop competition period on weeds and yield components of sesame (Sesamum indicum L.). Pakistan Journal of Weed Science Research, 17(1), 51-63.

Imoloame, E.O., Joshua, S.D., Gworgwor, N.A. (2010). Evaluation of post-emergence herbicides for weed control in sesame (Sesamum indicum) in sudan savanna zone of Nigeria. Nigerian Journal of Weed Science, 23(1), 73-85.

Langham, D.R., Grichar, J., Dotray, P. Review of herbicide research on sesame (Sesamum indicum L.), 2007. Disponível

https://www.researchgate.net/profile/Derald Langham/publication/268372403 REVIEW OF HERBICIDE RE SEARCH ON SESAME Sesamum indicum L/links/54bd1f240cf218da93918d7e/REVIEW-OF-HERBICIDERESEARCH-ON-SESAME-Sesamum-indicum-L.pdf. Acesso em: 21 abril 2020.

Lins, H.A., Souza, M.F., Albuquerque, J.R.T., Santos, M.G., Barros Júnior, A.P., Silva, D.V. (2019). Weed interference periods in sesame crop. Ciência e Agrotecnologia, 43(1), 1-10. https://doi.org/10.1590/1413$\underline{7054201943000819}$ 
Magani, E.I., Shave, P.A., Avav, T. (2012). Evaluation of fluazifop-p-butyl and propanil for weed control in sesame (Sesamum indicum L.) in Southern Guinea Savanna, Nigeria. Journal of Experimental Agriculture International, 2(4), 680-689. https://doi.org/10.9734/AJEA/2012/1606

Mane, S.V., Kanade, V.M., Shendage, G.B., Sarawale, P.P., Shetye, V.N. (2017). Weed management in sesamum (Sesamum indicum L.) grown under coastal region of Maharashtra. Journal of the Indian Society of Coastal Agricultural Research, 35(1), 31-33.

Mruthul, T., Halepyati, A.S., Chittapur, B.M. (2015). Chemical weed management in sesame (Sesamum indicum L.). Karnataka Journal of Agricultural Sciences, 28(2), 151-154.

Pereira, L.S., Souza, G.D., Oliveira, G.S., Silva, J.N., Costa, E.M., Ventura, M.V.A., Jakelaitis, A. (2020). Eficiência de herbicidas aplicados em pós-emergência na cultura do feijão-caupi. Colloquium Agrariae, 16(1), 29-42. https://doi.org/10.5747/ca.2020.v16.n1.a345

Perin, A., Cruvinel, D.J., Silva, J.W.S. (2010). Desempenho do gergelim em função da adubação NPK e do nível de fertilidade do solo. Acta Scientiarum Agronomy, 32(1), 93-98. https://doi.org/10.4025/actasciagron.v32i1.252110.4025/actasciagron.v32i1.2521

Queiroga, V.P., Freire, R.M.M., Firmino, P.T., Marinho, D.R.F., Silva, A.C., Barbosa, W.T., Queiroga, D.A.N. (2012). Avaliação da qualidade das sementes de gergelim submetidas aos processos de despeliculação manual, físico e mecânico. Revista Brasileira de Produtos Agroindustriais, 14(4), 307-315.

Ribeiro, S.B., Pagnocca, F.C., Victor, S.R., Bueno, O.C., Hebling, M.J., Bacci Júnior, M., Silva, O.A., Fernandes, J.B., Vieira, P.C., Silva, M.F.G.F. (1998). Activity of sesame leaf extracts against the symbiotic fungus of Atta sexdens L. Anais da Sociedade Entomológica do Brasil, 27(3), 421-426, 1998. http://dx.doi.org/10.1590/S0301$\underline{80591998000300010}$

Santos, H.G., Jacomine, P.K.T., Anjos, L.H.C., Oliveira, V.A., Lumbreras, J.F., Coelho, M.R., Almeida, J.A., Araujo Filho, J.C., Oliveira, J.B., Cunha, T.J.F. (2018). Sistema brasileiro de classificação de solos. Brasília: Embrapa.

Shaalan, A.M., Abou-Zied, K.A., El Nass, M.K. (2014). Productivity of sesame as influenced by weeds competition and determination of critical period of weed control. Alexandria Journal of Agricultural Research, 59(3), 179-187.

Silva, A.A., Silva, J.F., Ferreira, F.A., Ferreira, L.R., Silva, J.F. (1999). Controle de plantas daninhas. Viçosa: DFT/UFV.

Velini, E.D., Osipe, R., Gazziero, D.L.P. (1995). Procedimentos para instalação, avaliação e análise de experimentos com herbicidas. Londrina: SBCPD.

Witter, A.P.W., Nohatto, M.A., Borges, B.L., Kaseker, J.F., Rosa, E.F.F., Madeira, L.G., Fermiano, A.P. (2019). Fitossociologia e supressão de plantas daninhas sob efeito da solarização e cobertura com capim-elefante. Revista Brasileira de Agropecuária Sustentável, 9(1), 56-63. https://doi.org/10.21206/rbas.v9i1.3049 